\title{
Omar Badsha Rememberances of the Road to Harar
}

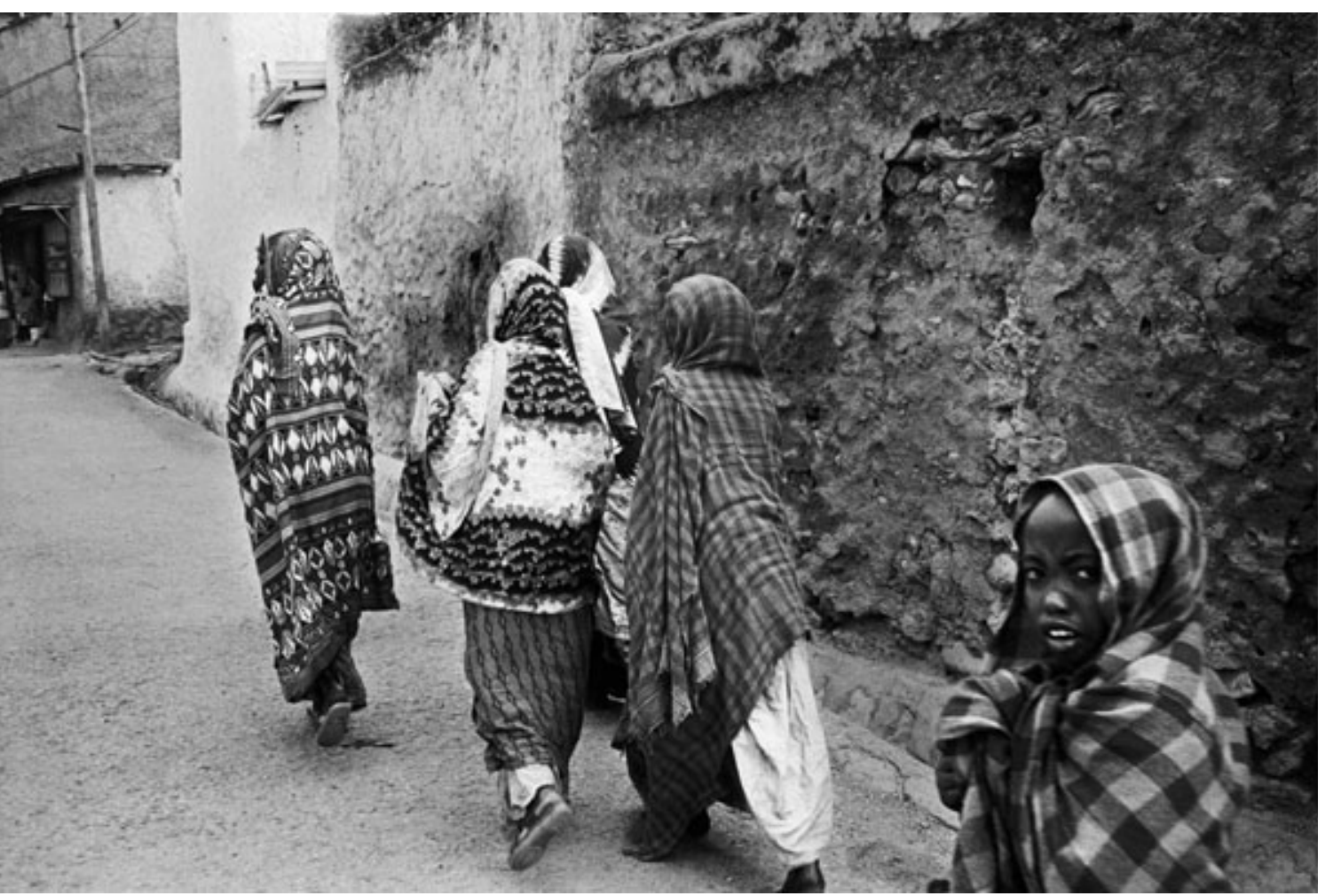

Omar Badsha is the founder and director of South African History Online, a website on history and culture. His photographic collections include Letter to Farzanah (1978), Imijondolo (1985), South Africa - The Cordoned Heart (1986), Imperial Ghetto (2001), With Our Own Hands (2002) and Amulets \& Dreams: War, Youth E Change in Africa (2002). Email: omar@sahistory.org.za 


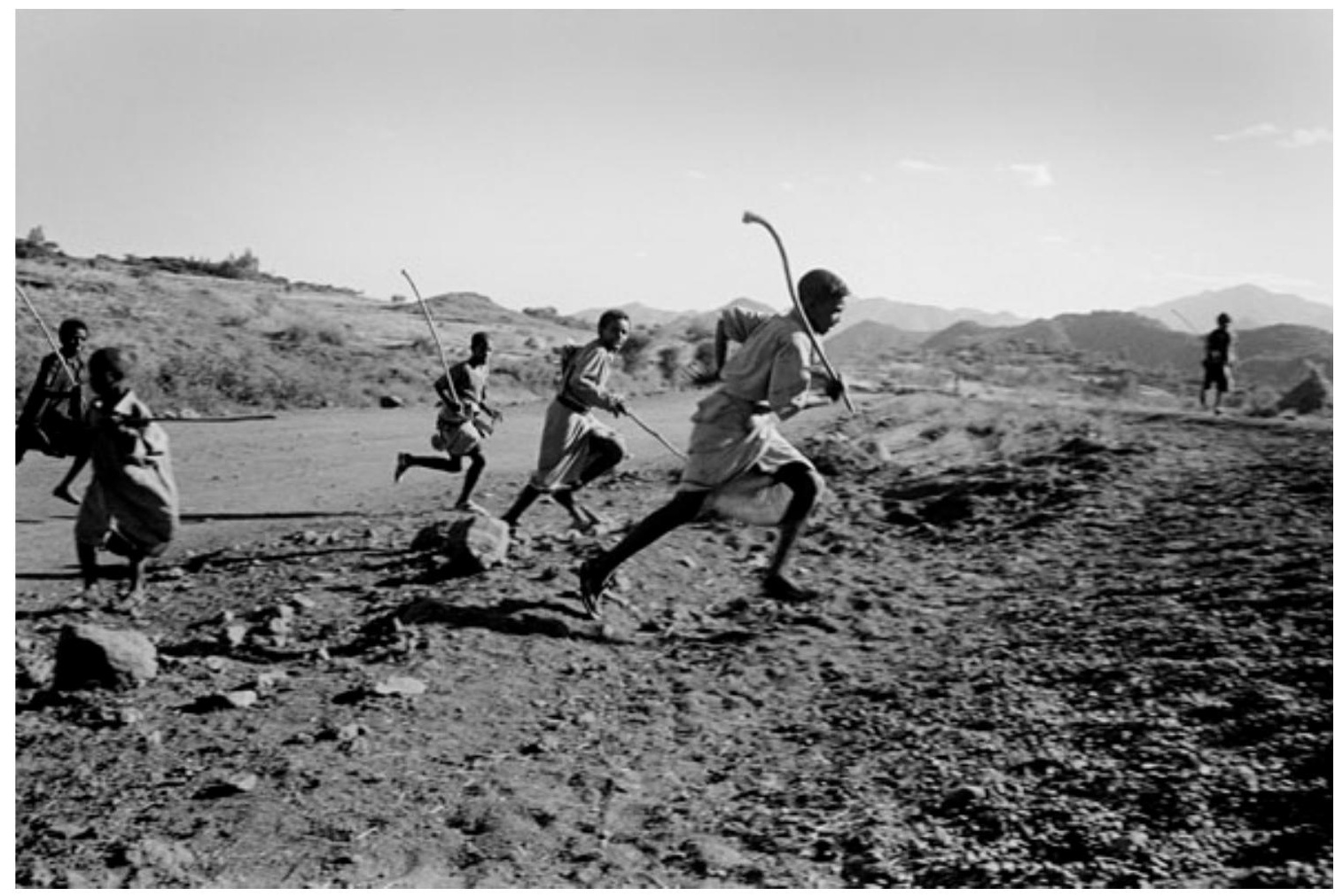




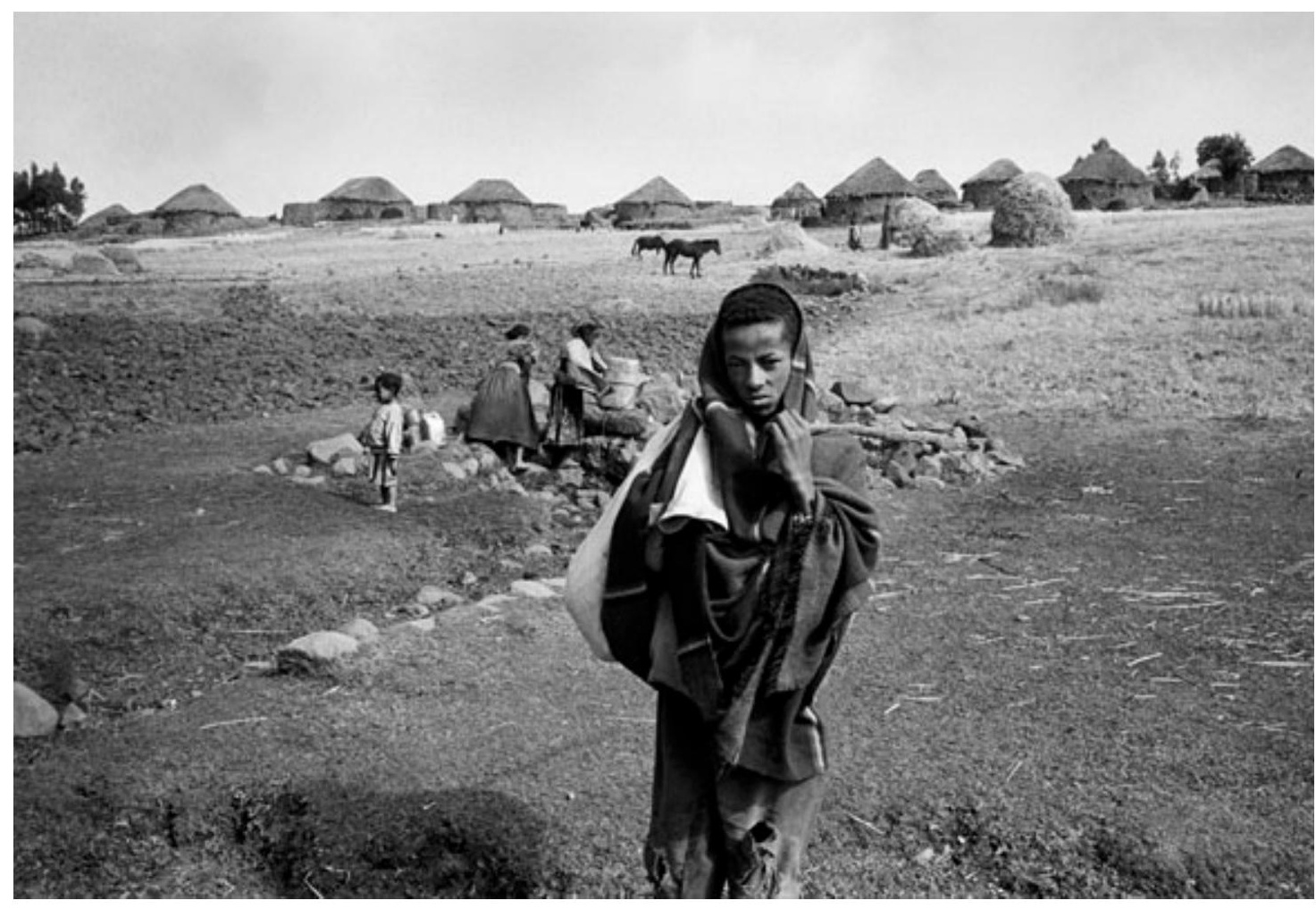


The photographs in this essay were taken on two occasions in 2001 and 2002. I visited Ethiopia to photograph the effects of war on children and the youth for a book that I was editing. On both occasions I visited the eastern town of Harar and lived in the old part of the city. I was struck by the impact that war, democratization and globalization had on the region, the people, their children and their culture. The changes in Harar epitomize similar happenings in the rest of Ethiopia and other third world countries I have visited. The subsequent book Amulets and Dreams: War, Youth and Change in Africa (SAHO, ISS, Unisa, 2002) and an accompanying travelling exhibition (see www.sahistory.org.za) look at war and its consequences in six African countries: Angola, Burundi, Eritrea, Ethiopia, Mozambique, and Sierra Leone.

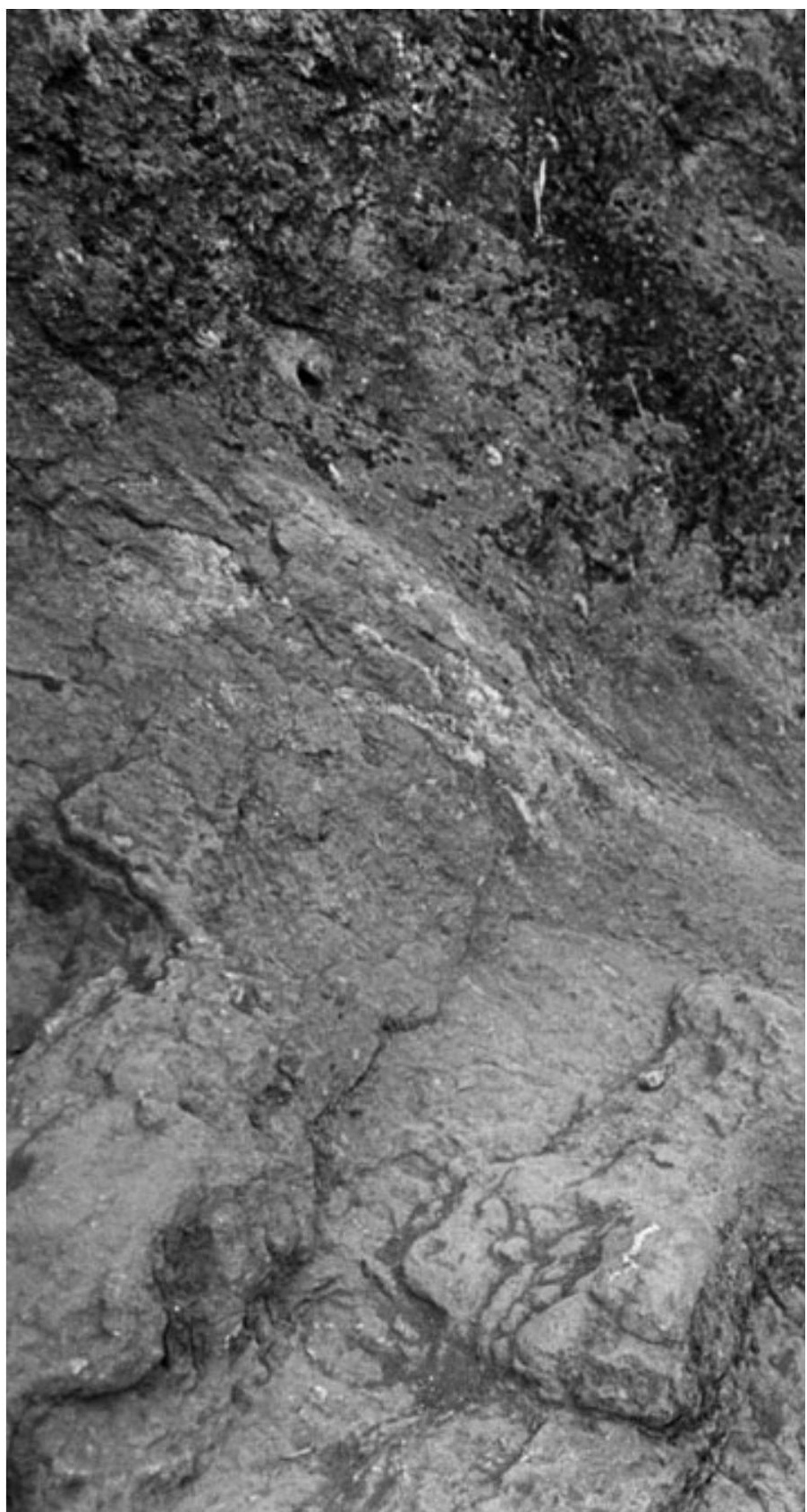




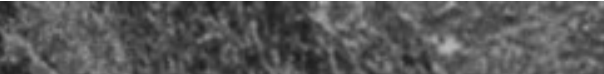

3.

Wh.

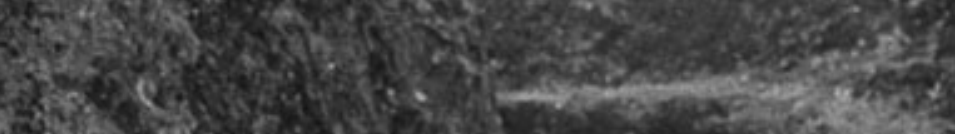

5.

S.3.

W.t.

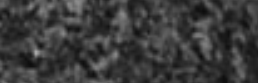

sine

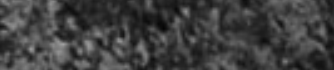

sots 15

(2)

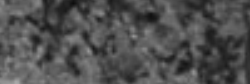

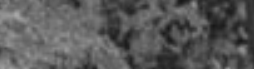

int $x, 30^{2}$

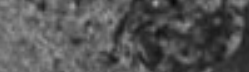

cos.

(t)

ixing

$2+3025$

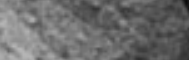

quisters

$2+53$

$\cos _{4} \times \mathrm{c}^{2}$

cosing

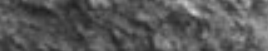

\section{(2) \\ 8} 1)

(2) $1 x^{2}=5$ $2 x^{2}=5.04$
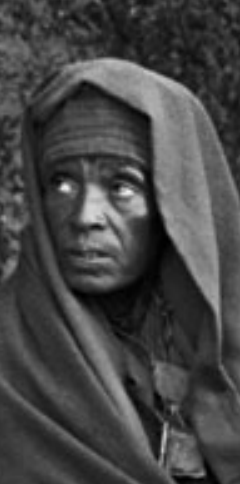

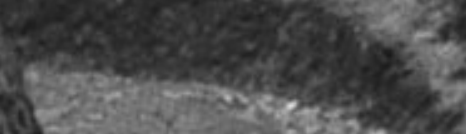

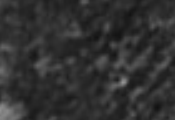

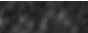

